\title{
Adesão a farmacoterapia em pacientes cadastrados no Hiperdia em uma Unidade Básica de Saúde em Aracaju, SE
}

\author{
Pharmacotherapy among patients from \\ Health Service in Aracaju, SE, Brazil
}

Recebido em: 11/08/2014 Aceito em: 25/09/2014
Kathlyn Pinheiro dos SANTOS ${ }^{1}$; Monia Carla Machado COSTA $^{1}$; Marcos Cardoso RIOS ${ }^{2}$; Priscila Souza de Sena RIOS $^{1}$

${ }^{1}$ Universidade Tiradentes, UNIT. Av. Murilo Dantas, 300. Farolândia, Aracaju, Sergipe, Brasil. CEP 49032-490; ${ }^{2}$ Universidade Feral de Sergipe. Campus São Cristóvão. Cidade Universitária Prof. José Aloisio de Campos. Av. Mal. Rondon, s/n. Jardim Rosa Elze. São Cristóvão, Sergipe, Brasil. CEP 49100-000.

\section{ABSTRACT}

Some of the complications of Hypertension and Diabetes mellitus could be preventable with primary care services and changes on patients' diet habit. Aiming to reduce the problems associated with the pharmacotherapy, Hiperdiais a good strategy. However, compliances problems still happened. The objective of the study was to evaluate the degree of adherence to pharmacotherapy and knowledge of patients with hypertension and type 2 diabetes enrolled in a Basic Health Unit in Aracaju, SE. Patients with hypertension and diabetes mellitus have been showed the same rate (79\%) of hits and they were classified according Batalla test as doers of drug treatment. Another key to understand the factors that associate low degree of membership aspect are the analysis of the Morisky and Green test. Following to the test, only $15 \%$ of patients were classified as adherent to medication treatment. Most significant results were from patients who had low level of compliance, resembled both intentions profile such as educational factors. Summarizing, will be necessary to create appropriate educational strategies for these groups to clarify the Hypertension and Diabetes mellitus

Keyword: Sadhesion; high blood pressure; Diabetes mellitus

\section{RESUMO}

Estudos têm demonstrado que algumas das complicações da Hipertensão Arterial e da Diabetes mellitus poderiam ser evitadas com os serviços de atenção básica e mudança de atitude do paciente. Com vistas à diminuição dos problemas associados à farmacoterapia, o Hiperdia aparece como ferramenta estratégica. Ainda assim, os problemas de adesão acontecem.O objetivo do estudo foi verificar o grau de adesão à farmacoterapia e conhecimento dos pacientes portadores de Hipertensão e Diabetes mellitus tipo 2 atendidos em uma Unidade Básica de Saúde em Aracaju, SE. Pacientes com hipertensão e diabetes mellitus apresentaram o mesmo índice (79\%) de acertos e por isso foram classificados, segundo teste de Batalla, como cumpridores do tratamento medicamentoso. Outro aspecto importante para o entendimento dos fatores que associam o baixo grau de adesão foia análise do teste de Morisky e Green. De acordo com o teste, apenas $15 \%$ dos pacientes foram classificados como aderentes ao tratamento medicamentoso. Resultados mais expressivos foram de pacientes que apresentaram baixo grau de adesão, com perfil semelhante ao de ambas as intencionalidades. Como os fatores educacionais estão envolvidos, torna-se necessário que estratégias educativas adequadas a estes grupos sejam realizadas, visando o esclarecimento sobre Hipertensão Arterial e Diabetes mellitus.

Palavras-Chave: adesão; hipertensão arterial; Diabetes mellitus 


\section{INTRODUÇÃO}

A Hipertensão Arterial Sistêmica (HAS) e a Diabetes mellitus (DM) apresentam altas prevalências e são responsáveis pelas maiores taxas de morbimortalidade da população brasileira e de todo mundo, gerando um alto custo social e financeiro $(1,2)$. Estudos têm mostrado que doenças crônicas, como HAS e DM, elevam os custos hospitalares por complicações clínicas dos casos e o tempo de internamento $(3,4,5)$. No Brasil, o custo anual avaliado no sistema público de saúde para o tratamento da hipertensão arterial, por exemplo, foi de aproximadamente US\$ 400 milhões na primeira década do século XXI e representou $1,43 \%$ dos gastos totais do SUS, sendo que algumas das complicações poderiam ser evitadas com os serviços de atenção básica e mudança de atitude do paciente (6).

Com vistas à diminuição dos problemas associados à farmacoterapia, o Programa Nacional de Hipertensão e Diabetes mellitus - Hiperdia é estratégico. O Hiperdia foi criado pelo Ministério da Saúde e está inserido no contexto do Programa Saúde da Família (PSF). Este programa permite cadastrar e acompanhar os portadores de HAS e/ou DM e possibilita detectar os fatores que diminuem o grau de adesão ao tratamento (7-11).

O conceito da adesão ao tratamento incorpora fatores sociais, econômicos e de comportamento, incluindo os associados à equipe de saúde e dos pacientes. Nesse sentido, ter o medicamento não é mais importante que usá-lo adequadamente (12). Estudos têm mostrado que a não adesão é comum em pacientes com doenças crônicas, incluindo a HAS e o DM, mesmo quando estes medicamentos estão disponíveis. Os índices variaram de $34 \%$ a $86 \%$, conforme a região e a doença, denotando terapias com baixo grau de adesão (10,13-16).

Um dos fatores associados ao baixo grau de adesão é a falta de conhecimento a respeito da doença, que, por exemplo, levou um grupo de pacientes a crer que estava curado e $16,28 \%$, abandonaram o tratamento (17). No estudo de Ceretta et al. (2009) o desconhecimento sobre a doença foi motivo pelo qual $8 \%$ dos entrevistados foram classificados como não-aderente ao tratamento, ficando atrás do fato de que estes pacientes não receberam explicações sobre a importância do uso adequado e contínuo da medicação (16).

De acordo com Chaui (1997, apud Rolim, 2008) o conhecimento é realizado como reflexão do entendimento, compreendendo que o sujeito do conhecimento é um ser racional consciente (18). Assim, é preciso que o paciente tenha e entenda as informações necessárias sobre sua saúde e medicamentos, de forma que possa assimilá-las, uma vez que falta de compreensão vem se constituindo numa das causas de insegurança do cliente ou até mesmo abandono do tratamento, de forma que a não adesão ou não cumprimento das metas farmacoterapêuticas pode associar-se ao nível de conhecimento do paciente sobre a doença e o tratamento proposto (19-21). Outros motivos destacados na literatura são o esquecimento, descuido e/ou mesmo a intenção (22).

Conhecer os padrões de utilização de medicamentos e conhecimento das doenças, em pacientes cadastrados no programa Hiperdia é essencial para avaliar o impacto das intervenções, planejar e estabelecer melhorias nos serviços de saúde. Mediante o exposto, o objetivo do presente trabalho foi avaliar o grau de adesão aos medicamentos para hipertensão e/ou diabetes mellitus e o conhecimento sobre a(s) doença(s)- hipertensão e/ ou diabetes mellitus- em pacientes cadastrados no programa Hiperdia em uma Unidade Básica de Saúde do município em Aracaju, SE.

\section{MÉTODOS}

A pesquisa foi do tipo exploratório com amostra não probabilística (conveniência). O estudo foi realizado em uma unidade básica de saúde no município de Aracaju, no período compreendido entre agosto a outubro de 2012 e foi aprovado pelo Comitê de Ética e Pesquisa da Universidade Tiradentes com número do protocolo 060512.

A amostra foi constituída por indivíduos com idade igual ou superior de 18 anos, diagnosticados com hipertensão arterial e/ou diabetes mellitus tipo 2, cadastrados no programa Hiperdia da unidade, fazendo terapia medicamentosa para pelo uma dessas condições e que concordaram em participar do estudo por meio da assinatura do Termo de Consentimento Livre e Esclarecido (TCLE).

Após a assinatura do TCLE, os voluntários foram entrevistados pelos pesquisadores para a coleta de informações referentes às variáveis sócio-demográficas e avaliação do grau de adesão à farmacoterapia, por meio da análise dos medicamentos utilizados e o comportamento admitido em relação ao uso desses medicamentos, definido pelo teste de Morisky e Green $(23,24)$. Foi também aplicado o método para avaliar o conhecimento do paciente sobre a(s) doença(s) que o acometia, por meio do teste de Batalla $(25,26)$. Os resultados foram analisados de maneira independente, mas estruturados em um formulário adaptado $(19,22,27,28)$.

De acordo com o protocolo do teste de Morisky e Green, foi considerado aderente ao tratamento o paciente que obteve pontuação máxima de quatro pontos (quando a resposta a todas as perguntas são negativas) e não ade- 
rente o que obteve três pontos ou menos, ou seja, pelo menos uma das respostas foi afirmativa $(23,24)$. Esta avaliação permitiu, também, discriminar se o comportamento de baixo grau de adesão foi do tipo intencional ou não intencional, sendo, também possível caracterizar usuários portadores de ambos os tipos de comportamentos de baixa adesão. Ao final de cada entrevista, todos os voluntários foram esclarecidos da importância da adesão aos medicamentos.

O Teste de Batalla, que inicialmente havia sido criado para avaliar o conhecimento sobre a HAS (25), foi adaptado para o DM 2(19), e devido a elevada sensibilidade e valor preditivo positivo, foi utilizado na presente pesquisa. O referido teste envolveu três perguntas para cada uma das possíveis doenças analisadas: Aos pacientes hipertensos foi perguntado: 1. A HAS é uma enfermidade para toda a vida? 2. A HAS pode ser controlada com dieta e medicação? 3. Cite dois ou mais órgãos que podem ser atingidos pela HAS.

Para os pacientes diagnosticados com diabéticos tipo 2, foi indagado: 1. A DM 2 é uma enfermidade para toda a vida? 2. A DM 2 pode ser controlada com dieta e medicação? 3. Cite dois ou mais órgãos que podem ser atingidos pela DM 2. A alguns pacientes foram feitas as perguntas relativas às duas condições clínicas, conforme prevalência e diagnóstico médico. Respostas corretas às questões relativas ao Teste de Batalla $(19,25,26)$ fornecem uma noção do conhecimento sobre a doença, fator relevante no processo de adesão à farmacoterapia.

As entrevistas foram realizadas por meio de visitas técnicas dos pesquisadores às residências dos pacientes cadastrados/acompanhados, juntamente com o Agente Comunitário de Saúde (ACS) da área analisada (nos dias agendados das visitas à comunidade), ou mesmo durante a busca pelos serviços médicos ou da farmácia nos dias de consulta e/ou recebimento dos medicamentos. Foram tomados cuidados preventivos para que o paciente não tivesse sido entrevistado mais de uma vez, necessitando o entrevistador anotar dados específicos adicionais do paciente entrevistado, como o endereço (área, microárea) e telefone, para quaisquer necessidades de intervenções presente e futura e evitar vieses.

Posteriormente os dados foram tabulados em planilha Excel. As análises estatísticas foram feitas utilizando o programa estatístico Prisma (Versão 5.01).

\section{RESULTADOS E DISCUSSÃO}

Foram entrevistados na presente pesquisa 276 pacientes cadastrados no programa Hiperdia da UBS analisada. No que se refere à prevalência da doença foi ob- servado que 261 indivíduos tinham diagnóstico de HAS, 73 eram diagnosticados com DM e 58 pacientes apresentavam as duas doenças (HAS e DM). Na Tabela 1 são descritas as principais características sócio-econômicas analisadas no grupo de entrevistados.

Tabela 1. Características sócio-econômicas de pacientes atendidos pelo Programa Hiperdia de uma Unidade Básica de Saúde de Aracaju,SE (agostooutubro, 2012).

\begin{tabular}{|c|c|}
\hline Variável & $\%$ \\
\hline \multicolumn{2}{|c|}{ Faixa Etária } \\
\hline 31- 40 anos & 4 \\
\hline 41- 60 anos & 53 \\
\hline $61-80$ anos & 40 \\
\hline$\geq 81$ & 3 \\
\hline Total & 100 \\
\hline \multicolumn{2}{|c|}{ Escolaridade } \\
\hline Analfabeto & 12 \\
\hline Ensino Fundamental Incompleto & 41,3 \\
\hline Ensino Fundamental Completo & 40,4 \\
\hline Nível Superior Incompleto & 0,8 \\
\hline Nível Superior Completo & 5,5 \\
\hline Total & 100 \\
\hline \multicolumn{2}{|c|}{ Estado Civil } \\
\hline Solteiro & 17 \\
\hline Casado & 48 \\
\hline União Consensual & 1 \\
\hline Separado/Divorciado & 20 \\
\hline Viúvo & 14 \\
\hline Total & 100 \\
\hline \multicolumn{2}{|c|}{ Ocupação } \\
\hline Do lar & 30 \\
\hline Aposentado & 39 \\
\hline Com vínculo empregatício & 20 \\
\hline Autônomo & 8 \\
\hline Desempregado & 2 \\
\hline Afastado & 1 \\
\hline Total & 100 \\
\hline \multicolumn{2}{|c|}{ Renda per capita } \\
\hline$\leq 1$ salário mínimo & 52 \\
\hline 1-2 salários mínimos & 27 \\
\hline 2-3 salários mínimos & 9 \\
\hline 4-5 salários mínimos & 6 \\
\hline$>5$ salários mínimos & 6 \\
\hline Ignorado & -- \\
\hline Total & 100 \\
\hline
\end{tabular}

Quanto ao acesso aos medicamentos, 41,2\% dos entrevistados relataram que os adquiriram integralmente gratuito pelo Sistema Público de Saúde (SPS); 29,3\% re- 
lataram que receberam parte dos medicamentos do SPS e outra parte teve que adquirir com recursos próprios; 9,5\% destacaram que a obtenção foi feita integralmente com recursos próprios ou foram parcialmente obtidos de entidades não governamentais (doações, entre outras) (20\%). Quando não disponíveis no SPS, 96,5\% dos pacientes entrevistados na presente pesquisa afirmaram que compravam e 3,5\% afirmaram que não utilizavam o medicamento.

Os medicamentos mais utilizados entre os participantes do estudo foram o anti-hipertensivo captopril $(32,5 \%)$ e o hipoglicemiante metformina (17\%). Cerca de $10 \%$ dos pacientes não souberam responder quais medicamentos utilizavam naquele momento. Entre outros medicamentos citados estavam também o hidroclorotiazida e a glibenclamida (Tabela 2).

De acordo com o teste de Morisky e Green (24), 15\% dos pacientes foram classificados como aderentes ao tratamento medicamentoso, ou seja, responderam negativamente a todas as perguntas referentes ao teste (Tabela 3).
Tabela 2. Medicamentos mais prevalentes entre os usuários atendidos pelo Programa Hiperdia de uma Unidade Básica de Saúde de Aracaju,SE (agostooutubro, 2012).

\begin{tabular}{|l|c|c|}
\hline Indicação Clínica & Medicamento & $\%$ \\
\hline \multirow{2}{*}{ Hipertensão } & captopril & 32,5 \\
\hline \multirow{2}{*}{ Diabetes mellitus } & hidroclorotiazida & 21,40 \\
\hline & Outros & 46,1 \\
\hline & glibenclamida & 12 \\
\hline & metformina & 17 \\
\hline
\end{tabular}

Respostas positivas às primeiras duas perguntas caracterizaram a não intencionalidade do comportamento. Já as perguntas $3 \mathrm{e} 4$, permitiram classificar os pacientes com comportamento intencional frente a farmacoterapia. Dessa forma, para ambas as condições, a não adesão não intencional ocorreu principalmente devido ao esquecimento e de forma intencional, quando os pacientes se sentiam bem.

Tabela 3. Avaliação da adesão ao tratamento medicamentoso de usuários atendidos pelo Programa Hiperdia em uma Unidade Básica de Saúde em Aracaju, SE, no ano de 2012, segundo o teste de Morisky e Green (1986).

\begin{tabular}{|c|c|c|c|c|}
\hline \multirow{3}{*}{ Questões } & \multicolumn{4}{|c|}{ Classificação } \\
\hline & \multicolumn{2}{|c|}{ Hipertensão } & \multicolumn{2}{|c|}{$\begin{array}{l}\text { Diabetes } \\
\text { mellitus }\end{array}$} \\
\hline & Não & $\operatorname{Sim}$ & Não & Sim \\
\hline 0 (a) senhor (a) alguma vez, esqueceu de tomar seus medicamentos? & 25,5 & 74,5 & 18,2 & 81,8 \\
\hline Ás vezes é descuidado (a) quanto ao horário de tomar seu medicamento? & 51,8 & 49,2 & 54,5 & 45,5 \\
\hline Quando (o) a senhor(a) se sentiu bem, alguma vez, você deixou de tomar seu medicamento? & 23,8 & 76,2 & 21,2 & 78,8 \\
\hline Quando se sente mal com o medicamento, às vezes, deixou de tomá-lo? & 39,0 & 51 & 30,3 & 69,7 \\
\hline
\end{tabular}

A investigação sobre o conhecimento da doença mostrou que parte expressiva dos entrevistados que possuíam HAS $(78,8 \%)$ tinham o conhecimento da cronicidade da doença e consequentemente da inexistência da cura. No que se refere à dieta, foi observado que $95,7 \%$ sabiam que a doença pode ser controlada com dieta e medicamentos. Quanto à questão subjetiva, visando avaliar o conhecimento do paciente sobre a gravidade/ especificidade da doença,35,5\% não souberam responder quais os órgãos afetados, e 27,3\% citaram cerca de 2 órgãos. Dentre os mais afetados pela doença o coração e os rins foram os mais citados.

Um perfil semelhante ao conhecimento do paciente com HAS foi observado com os pacientes com DM. Aproximadamente $79 \%$ dos pacientes também possuíam conhecimento quanto à cronicidade. Quanto ao controle da doença com dieta e medicamentos, aproximadamente 94\%sabiam que a doença pode ser controlada desta maneira. Ainda, $42 \%$ dos pacientes souberam informar pelo menos um órgão afetado, os quais foram mais citados, os olhos e os rins.

Os resultados mostraram que houve uma maior participação de pacientes do sexo feminino $(79,4 \%)$ corroborando com outros estudos $(29,30)$. Este fator pode ser atribuído a uma maior demanda e à utilização dos serviços de saúde por este grupo, que procura por assistência médica, em virtude de uma percepção mais acentuada das doenças (31). Esses números podem ainda apresentar uma relação com o predomínio deste gênero no cadastro do Hiperdia na UBS. A maior parte dos pacientes cadastrados no Hiperdia é do gênero feminino. Outro fator que pode estar associado à prevalência é a característica da pesquisa. Foram entrevistadas pessoas que convenientemente se dispusessem a responder às perguntas.

A idade prevalente, maior de 40 anos, tem influência na função cognitiva e visual do idoso, dificultando a distinção e a manipulação de diversos medicamentos ao mesmo tempo, o que pode comprometer o seguimento 
da terapêutica (32). Outra característica importante é relativa à escolaridade. Foi observado que $41,3 \%$ dos entrevistados possuíam o ensino fundamental incompleto, enquanto $12 \%$ eram analfabetos. Estefator pode ser uma barreira na compreensão das informações passadas pelo médico, especialmente em relação às medidas quanto ao tratamento correto (33).

O estado civil, a ocupação e a renda per capita também estão associados à adesão. Santos et al. (2013) associam a adesão ao contexto coletivo, visto que pessoas de um mesmo contexto social podem ajudar a enfrentar os diversos problemas, para além dos problemas de saúde (34). Dessa forma, ser casado pode influenciar positivamente a adesão. Indivíduos que compartilham experiências com seus cônjuges e recebem apoio deles podem ter melhor participação no seguimento do tratamento. Para Saraiva et al. (2007) a família assume uma parcela significativa de responsabilidade na prestação do cuidado à saúde de seus membros, especialmente em relação ao doente crônico (35). Aproximadamente 49\% dos pacientes entrevistados eram casados.

A renda per capita e a ocupação também são fatores capazes de influenciar a adesão à farmacoterapia, sendo maior o grau de adesão quanto maior for a renda $(36,37)$. A maior parte dos pacientes entrevistados na pesquisa era de aposentados (39\%) com renda per capita igual ou inferior a um salário mínimo (52\%). Silva et al. (2010) haviam discutido a renda como importante variável, uma vez que os idosos, a exemplo do presente estudo, apresentam renda insuficiente para arcar com as demandas pertinentes à idade; sobretudo, as demandas de saúde, incluindo os medicamentos (32).

Didier e Guimarães (2007) destacaram em seu estudo que a maior parte dos participantes faziam uso de dois anti-hipertensivos, sendo hidroclorotiazida e captopril, os mais usados acompanhados da nifedipina (38). Para os diabéticos, os mais utilizados foram as sulfoniluréias (glibenclamida ou glicazida), com uma pequena diferença em relação aos que faziam uso da metformina.

Como observado no presente estudo, em geral, estes medicamentos são adquiridos através do serviço público de saúde. No entanto, em determinadas situações, como o desabastecimento da UBS, é preciso adquirir com recursos próprios. Embora a compra direta, aquela realizada pelo próprio usuário, possa representar a ascensão econômica, a cronicidade da doença e o uso contínuo dos medicamentos podem ter impacto negativo na adesão (39). Na falta de disponibilidade de recursos, alguns pacientes acabam não fazendo uso do medicamento.
O baixo grau de adesão em pacientes com doenças crônicas já é bem destacado na literatura $(11,19,40)$. A análise do teste de Morisky e Green (24) permitiu observar que apenas $15 \%$ dos pacientes foram classificados como aderentes ao tratamento medicamentoso. Resultados mais expressivos foram de pacientes que apresentaram baixo grau de adesão, com perfil semelhante ao de ambas às intencionalidades.

Para ambas as condições clínicas, o principal motivo, de acordo com os pacientes, foi o esquecimento. Os números apresentados no estudo são mais expressivos do que os apresentados por Rocha et al. (2007), cujo item foi relatado por $33,4 \%$ dos entrevistados, seguidos por problemas referentes ao descuido com os horários de administração (25\%), interrupção do tratamento pela melhora dos sintomas $(23,3 \%)$ e aumento da quantidade de fármacos pela piora dos sintomas (26,4\%) (27). Outros estudos também já referiram o esquecimento e o descuido como os principais problemas da não adesão $(22,41)$.

O teste de Batalla (25) possibilitou identificar outros problemas associados. A falta de conhecimento sobre os riscos da doença pode ser um dos motivos do abandono do tratamento. Um dos indícios do problema foi a afirmação feita por mais de $20 \%$ dos pacientes que alegaram interromper a medicação quando se sentiam bem. Estudos têm mostrado que quando o paciente sabe a importância do tratamento ele se sente encorajado a decidir a manutenção da farmacoterapia, frente a efeitos colaterais menores $(19,25)$. Para isso é preciso estimular o paciente a exercer o controle de sua(s) doença(s) (42). Outro problema evidente são os efeitos colaterais, que segundo Cintra et al. (2010), reduzem em até sete vezes as chances de adesão ao tratamento (43). Esses problemas foram destacados por $39 \%$ dos pacientes com HAS e $30,3 \%$ dos pacientes acometidos pela DM.

A falta de conhecimento também é apontada em outros estudos, como fatores associados ao baixo grau de adesão. Segundo Carvalho et al. (2012) cerca de $73 \%$ dos entrevistados responderam corretamente à primeira pergunta e aproximadamente $98 \%$ a segunda; contudo, poucos souberam responder os órgãos afetados $(33,3 \%)$, e os mais citados pelos entrevistados foram o coração e o cérebro (11). Araújo et al. (2011) haviam observado que $73,2 \%$ dos entrevistados tinham conhecimento da cronicidade do DM 2, e $87,9 \%$ deles entendiam que a doença pode ser controlada com dieta e medicamentos; quanto aos órgãos afetados $47,2 \%$ não souberam informar e $20,1 \%$ obtiveram apenas um acerto (19). 
O cerne do problema pode estar na relação profissional-paciente e educação, haja vista que o desconhecimento sobre as doenças foi um dos motivos de descumprimento. Quando o paciente não possui o conhecimento da doença, este tende a apresentar uma baixa adesão ao tratamento, ainda que a obtenção gratuita do medicamento sirva como uma alternativa para favorecer a adesão (11). É preciso investir na qualidade do atendimento e evitar ruídos de comunicação. Neste sentido, o profissional de saúde, entre os quais o farmacêutico, deve adotar uma postura pró-ativa na transmissão de informações aos pacientes, permitindo que este perceba que pode controlar os sintomas com atitudes de maior adesão $(44,45)$.

\section{CONCLUSÃO}

A evidência de que boa parte dos pacientes entrevistados apresentava pouco conhecimento sobre a existência de cura ou entendimento do controle com dieta e medicamentos pode ser associada à baixa adesão farmacoterapêutica. A falta de conhecimento sobre os riscos da doença também é apontado como um dos motivos do abandono do tratamento. Um dos indícios do problema é a afirmação feita pelos pacientes de que paravam de administrar seus medicamentos quando se sentiam bem. Os resultados sugerem a necessidade de campanhas educativas, nas quais os profissionais de saúde adotem uma postura ativa na transmissão de informação.

\section{REFERÊNCIAS}

1. BRASIL. Ministério da Saúde (MS). Vigitel Brasil 2009: vigilância de fatores de risco e proteção para doenças crônicas por inquérito telefônico. Brasília: MS; 2010.

2. Castro NG, Cunha ER, Santos MDS, Dias RS. Hiperdia: conhecimento da cobertura do programa no Maranhão. Cad. Pesq. 2010, 17(2): 77-83.

3. Araújo DV, Tavares LR, Verrísimo R, Ferraz MB, Mesquita ET. Custo da insuficiência cardíaca no Sistema Único de Saúde. Arq Bras Cardiol. 2005, 84 (5): 422-427. DOI: 10.1590/S0066-782X2005000500013.

4. Ribeiro RA, Mello RGB, Melchior R, Dill JC, Hohmann CB, Lucchese AM. Custo anual do manejo da cardiopatia isquêmica crônica no Brasil. Perspectiva Pública e Privada. Arq Bras Cardiol. 2005, 85 (1): 3-8. DOI: 10.1590/ S0066-782X2005001400002.

5. Silva WCM, Farah BQ, Ricarte GB. Atividade Física e Fatores Associados em Usuários do Programa Hiperdia de uma Unidade de Saúde da Família do Recife. Rev Bras Cien Saúde. 2012, 16(3):385-392.

6. Dib MW, Riera R, Ferraz MB. Estimated annual cost of arterial hypertension treatment in Brazil. Rev Panam Salud Publica. 2010, 27 (2): 125-131.

7. BRASIL. Ministério da saúde. DATASUS - Departamento de Informática do SUS, 2008.Disponível em: http:// www2.datasus.gov.br/ DATASUS/index.php?area=0403. Acesso em: 10 agosto 2012.

8. Souza MLP, Garnelo L. "É muito dificultoso!": etnografia dos cuidados a pacientes com hipertensão e/ou diabetes na atenção básica, em Manaus, Amazonas; Brasil. Cad Saúde Publica. 2008, 24 (1): 91-99. DOI:10.1590/S0102$-311 X 2008001300014$.

9. Miranzi SSC, Ferreira FS, Iwamoto HH, Pereira GA, Miranzi MAS. Qualidade de Vida de Indivíduos com Diabetes Mellitus e Hipertensão acompanhados por uma equipe de Saúde da Família. Texto \& Contexto - Enferm. 2008, 17(4): 672-679. DOI: 10.1590/S0104-07072008000400007.
10. Gomes TJO, Silva MVR, Santos AA. Controle da pressão arterial em pacientes atendidos pelo programa Hiperdia em uma Unidade de Saúde da Família. Rev Bras Hipertens. 2010, 17(3): 132-139.

11. Carvalho ALM, Duarte Leopoldino RW, Silva JEG, Cunha CP. Adesão ao tratamento medicamentoso em usuários cadastrados no Programa Hiperdia no município de Teresina (PI). Cien Saúde Coletiva. 2012 , 17(7): 1885-1892. DOI: $10.1590 /$ S141381232012000700028 .

12. WHO. Adherence to long-term therapies: evidence for action. Geneva: World Health Organization. 2003.

13. Girotto E. Adesão ao tratamento anti-hipertensivo e fatores associados na área de abrangência de uma Unidade de Saúde da Família, Londrina, PR.; Dissertação (Mestrado em Saúde Coletiva) - Universidade Estadual de Londrina, Centro de Ciências da Saúde, Programa de Pós-Graduação em Saúde Coletiva, 2008.

14. Pires GB, Freitas IV. Prevalência de adesão ao tratamento farmacológico hipoglicemiante. Sitientibus. 2006, 34:37-45.

15. Dosse C, Cesarino CB, Martin JFV, Castedo MCA. Fatores associados à não adesão dos pacientes ao tratamento de Hipertensão Arterial.. Rev Latino-am Enfermagem, março-abril. 2009, 17(2):1-7.

16. Ceretta LB, Schwalm MT, Bez B, Ioná V, Macedo S, Ceretta RA. Adesão do Diabético ao tratamento farmacológico: Implicações para a Enfermagem. In: $61^{\circ}$ Congresso Brasileiro de Enfermagem. Fortaleza- CE, 2009.

17. Chamorro MAR, Chamorro AR, Jiménez EG. Incumplimiento terapéutico en pacientes en Seguimiento Farmacoterapéutico mediante el método Dáder en dos farmacias rurales. Pharm Care Esp. 2006, 8(2):62-68.

18. Rolim RA. Atenção farmacêutica: Um processo educativo. Infarma, 2008, 20:3-4. 
19. Araujo MFM, Freitas RWJF, Fragoso LVC, Araújo TM, Damasceno MMC, Zanetti ML. Cumprimento da terapia com antidiabéticos orais em usuários da atenção primária. Texto Contexto Enferm. 2011, 20 (1):135-43.

20. Alvim NAT, Ferreira MA. Perspectiva problematizadora da educação popular em saúde e a enfermagem. Texto Contexto Enferm. 2007, 16(2):315-319.

21. Rubin RR. Adherence to pharmacologic therapy in patients with type 2 diabetes mellitus. Am J Med. 2005, 118(5A):27-34.

22. Dewulf NLS, Monteiro RA, Passos ADC, Vieira EM, Troncon LEA. Adesão ao tratamento medicamentoso em pacientes com doenças gastrointestinais crônicas acompanhados no ambulatório de um hospital universitário. Rev Bras Ciênc Farm. 2006, 42 (4): 575-584.

23. Morisky DE, Levine M, Green LW, Smith CR. Health education program effects on the management of hypertension in the elderly. Arch. Intern. Med., 1982, 142 (10): 1835-1838.

24. Morisky DE, Green LW, Levine DM. Concurrent and predective validity of self-reported measure of medication adherence. Med. Care. 1986, 24: 67-74.

25. Batalla C, Blanquer A, Ciurana R, Garcia M, Cases E, Pérez A .Cumplimiento da lá prescripción farmacológica em pacientes hipertensos. Atencão Primária. Ene. 1994, 11: $185-91$.

26. Carvalho MC, Almeida APM, Garbinato LR. A assistência farmacêutica no atendimento aos pacientes do hiperdia do ESF 18 e 19 da cidade de Dourados/MS. Interbio. 2012, 6 (2): 5-15.

27. Rocha CH, Oliveira APS, Ferreira C, Faggiani FT, Schoeter G, Souza ACA, DeCarli GA, Morrone FB, Werlang MC. Adesão à prescrição médica em idosos de Porto Alegre, RS. Ciên Saúde Coletiva. 2008, 13(Sup):703-710.

28. Amarante LC, Shoji LS, Beijo LA, Lourenço EB, Marques LAM. A influência do acompanhamento farmacoterapêutico na adesão à terapia anti-hipertensiva e no grau de satisfação do paciente. Rev Ciênc Farm Básica Apl.2010, 31(3): 209-215.

29. Barros MBA, César CLG, Carandina L, Torre GD. Desigualdades sociais na prevalência de doenças crônicas no Brasil, PNAD 2003. Ciência \& Saúde Coletiva.2006, 11(4): 911-926. DOI: 10.1590/S1413-81232006000400014.

30. Bezerra DS, Silva AS, Carvalho ALM. Avaliação das características dos usuários com hipertensão arterial e/ou diabetes mellitus em uma Unidade de Saúde Pública, no município de Jaboatão dos Guararapes-PE, Brasil. Rev Ciênc Farm Básica Apl. 2009, 30 (1): 69-73.

31. Rocha CH, Oliveira APS, Ferreira, C, Faggiani FT, Schroeter G, Souza ACA, De Carli GA, Morrone FB, Werlang MC. Adesão à prescrição médica em idosos de Porto Alegre, RS, Brasil. Cien Saude Colet. 2008, 13(Sup):703-710.

32. Silva CSO, Pereira MI, Yoshitome AY, Rodrigues Neto JF, Barbosa DA. Avaliação do uso de medicamentos pela população idosa em Montes Claros, Minas Gerais, Brasil. Esc Anna Nery RevEnferm. 2010; 14(4):811-818
33. Cruzeta APS, Dourado, ACL, Monteiro MTM, Martins RO, Calegario TA, Galato D. Fatores associados à compreensão da prescrição médica no Sistema Único de Saúde de um município do Sul do Brasil. Cien Saude Colet. 2013, 18 (12): 3731-3737. DOI: 10.1590/S141381232013001200029 .

34. Santos ZMSA, Marques AC, Carvalho YP. Condições favoráveis à adesão ao tratamento. Rev Bras Promoc Saude. 2013, 26(2): 298-306.

35. Saraiva KRO, Santos ZMSA, Landim FLP, Lima HP, Sena VL. O processo de viver do familiar cuidador na adesão do usuário hipertenso ao tratamento. Texto Contexto Enferm, 2007;16(1):63-70

36. Marques PAC, Pierin AMG. Fatores que influenciam a adesão de pacientes com câncer à terapia antineoplásica oral. Acta Paul Enferm. 2008,21(2):323-329.

37. Musial DC, Dutra JS, Becker TC. A. A automedicação entre os brasileiros. SaBios - Revista de Saúde e Biologia, v. 2: 5-8, 2007. Apud Gonçalves D, Santos BRM, Gonçalves ML, Aragão CCV, Gatti RM, Yavo B. Prática de automedicação entre usuários de uma farmácia-escola. Rev Bras Ciên Saúde. 2009, 7(22): 23-32.

38. Didier MT, Guimarães AC. Otimização de Recursos no Cuidado Primário da Hipertensão Arterial. Arq Bras Cardiol. 2007, 88 (2): 218-224. DOI: $10.1590 /$ S0066$-782 X 2007000200014$.

39. Santos P, Costa RN, Castro. OCC. Quem acessa o programa farmácia Popular no Brasil? Aspectos do fornecimento público de medicamentos. Ciên Saúde Colet. 2011, 16 (6): 2963-2973.

40. Rios, MC, Carvalho RGB, Rios PSS. Avaliação da adesão farmacoterapêutica em pacientes atendidas em um programa assistencial ao idoso. Rev. Bras. Farm. 2014, 95 (1): $544-560$.

41. Dourado CS, Macêdo-Costa KNF, Oliveira JS, Leadebal ODCP, Silva GRF. Adesão ao tratamento de idosos com hipertensão em uma unidade básica de saúde de João Pessoa, Estado da Paraíba. Acta Scientiarum. Health Sciences. 2011, 33 (11): 9-17. DOI: 10.4025/actascihealthsci. v33i1.7708

42. Berger BA. Habilidade de comunicação para farmacêuticos: construindo relacionamentos, otimizando o cuidado aos pacientes./ Bruce A. Berger; tradução Divaldo Pereira de Lyra Junior et al. - São Paulo: Ed. Pharmabooks, 2011.

43. Cintra FA, Guariento ME, Miyasaki LA. Adesão medicamentosa em idosos em seguimento ambulatorial. Ciên Saúde Colet. 2010, 15(Supl. 3):3507-3515. DOI: 10.1590/ S1413-81232010000900025.

44. Possamai FP, Dacoreggio MS. A habilidade de comunicação com o paciente no processo de atenção farmacêutica. Trab. Educ. Saúde. 2008, 5: 473-490.

45. Silva EAC, Oliveira LM, Resende SG, Cardoso RA. Avaliação do impacto da dispensação orientada sobre a adesão farmacoterapêutica de pacientes hipertensos. Rev. Bras. Farm. 2008, 89(4): 315-331 